\title{
Modelling of Creep and Stress Relaxation Test of a Polypropylene Microfibre by Using Fraction-Exponential Kernel
}

\author{
Andrea Sorzia \\ Dipartimento di Scienze e Metodi dell'Ingegneria (DISMI), Università di Modena e Reggio Emilia, 42122 Reggio Emilia, Italy \\ Correspondence should be addressed to Andrea Sorzia; andrea.sorzia@unimore.it
}

Received 4 December 2015; Accepted 4 May 2016

Academic Editor: Julius Kaplunov

Copyright (c) 2016 Andrea Sorzia. This is an open access article distributed under the Creative Commons Attribution License, which permits unrestricted use, distribution, and reproduction in any medium, provided the original work is properly cited.

\begin{abstract}
A tensile test until breakage and a creep and relaxation test on a polypropylene fibre are carried out and the resulting creep and stress relaxation curves are fit by a model adopting a fraction-exponential kernel in the viscoelastic operator. The models using fractionexponential functions are simpler than the complex ones obtained from combination of dashpots and springs and, furthermore, are suitable for fitting experimental data with good approximation allowing, at the same time, obtaining inverse Laplace transform in closed form. Therefore, the viscoelastic response of polypropylene fibres can be modelled straightforwardly through analytical methods. Addition of polypropylene fibres greatly improves the tensile strength of composite materials with concrete matrix. The proposed analytical model can be employed for simulating the mechanical behaviour of composite materials with embedded viscoelastic fibres.
\end{abstract}

\section{Introduction}

Fibre-reinforced composite materials consist of fibres with high strength and elastic modulus embedded in a matrix to produce a combination of properties that cannot be achieved by single constituents. Usually, fibres are the principal loadcarrying members, while the surrounding matrix keeps them in the desired location and orientation. The matrix acts as a load transfer medium between fibres and plays a number of useful functions, for example, protecting the fibres from environmental damage. Fibres may be made up of various materials, such as steel, polymer, glass, or carbon, whereas polymer, metal, or ceramic can be chosen for the matrix material.

Fibre-reinforced polymer composites are probably the most important and widespread fibre-reinforced materials used for commercial and industrial applications. This is due to the combination of their low density, strength-weight ratios, and modulus-weight ratios that make them more attractive than many traditional metallic materials [1].

A classic example of fibre-reinforced composite used in civil engineering is Fibre-Reinforced Concrete (FRC), widely adopted for industrial pavements, tunnel linings, marine structures, earthquake-resistant structures, and plate and slab foundation [2]. In FRC composites, fibres are commonly added to concrete mixture in random disposition and short cuts in order to increase the cracking behaviour of concrete, thus transforming concrete from brittle into a ductile material [3]. However, there are composite materials in which fibres are aligned along particular directions, as it occurs in FRP frame elements (these composites can be studied by adopting, for instance, the approaches proposed in $[4,5])$.

Recently, the use of macro synthetic fibres made of polymeric materials has been proposed for structural purposes $[6,7]$. Experimental tests performed by Lanzoni et al. [8] show that the addition of polypropylene-based draw-wired fibres significantly improves crack resistance of the concrete mixture and enhances toughness and durability of FRC structural elements. Fibres could be found in interesting applications as an additive to improve concrete under aggressive environments (the mechanical behaviour has been studied under high thermal loads also $[9,10]$ and nuclear radiation [11]). However, particularly in such harsh conditions, a wide class of additives can be inserted at the mixing stage (like fly ash) to increase resistance of the concrete mixture [12].

Ductility and flexural strength of FRC are defined by energy-dissipation mechanisms during the pullout of the fibres that occurs in the opening propagating cracks $[13,14]$. 
Pullout of the fibres begins after cracking the concrete matrix (for the stress and strain localizations at the crack tip, see, e.g., [14-17]) and ceases with the complete extraction of the fibres and its evolution depends on the bonding between fibre and matrix $[18,19]$. Nevertheless due to their chemical inertness, polypropylene (PP) synthetic fibres have poor adhesion to the cement matrix with respect to other kinds of fibres. However, special surface nanotreatment can actually increase the adhesion properties between concrete and synthetic fibres, as shown by Di Maida et al. [3]. In this case, PP fibres may experience significant loading and consequently the viscous deformations of the composite material may considerably increase (for time-dependent effects in concrete structures see [20-22]). A single fibre can be studied as an embedded cylindrical body or a viscoelastic circular nanobeam in frictional contact with the cement matrix under axial tensile load (e.g., [23-25]).

Another application of synthetic fibres in civil engineering is the fibre-reinforced polymer (FRP) composites for reinforcement and retrofitting of concrete and masonry members, with applications in new buildings as well as for strengthening and/or rehabilitation of existing (prestressed as well as nonprestressed) structural members of both prefabricated and cast-in-place frames. FRP reinforcement consists in strengthening fibres applied to structural elements by a cementitious or polymeric-based layer. The mechanical performances of such systems can be assessed by solving the contact problem between two bounded layers [26-28] where the adhesive layer is a fibre-reinforced composite material [29] or, in a simpler way, as a fibre-reinforced Kirchhoff plate [30].

This work presents a creep and stress relaxation test performed over a PP synthetic fibre used for FRC. Creep is a time-dependent deformation of a viscoelastic material under the application of a constant stress at a constant temperature. Relaxation is the counterpart of creep, namely, a timedependent stress of a viscoelastic material under the application of a constant deformation at a constant temperature. Both are complex phenomena for they depend on material properties (e.g., molecular orientation and crystallinity) and external conditions (e.g., applied stress, temperature, and moisture). Moreover, the viscoelastic behaviour of PP fibres embedded in an elastic matrix complicates the modelling of creep and stress relaxation response of the composite material, which depends on many additional parameters such as concentration, aspect ratio, orientation, and, obviously, mechanical properties of PP fibres.

Creep and stress relaxation tests best demonstrate the viscoelastic characteristics of a polymeric solid. In creep test, a constant stress is maintained on a specimen while its deformation is monitored as a function of time, and deformation increases with time. In stress relaxation test, a constant deformation is maintained while the stress on the specimen is monitored as a function of time, and stress decreases with time. Typical creep and stress relaxation diagrams exhibit an instantaneous elastic response followed by a delayed time-dependent response [1].

Over the experimental creep and stress relaxation tests performed, this paper proposes an analytical model to fit these experimental curves. The model uses fractionexponential kernel in the viscoelastic operator and was proposed, for the first time, by Scott Blair and Coppen $[31,32]$ and Rabotnov [33] independently.

The classical viscoelastic constitutive models represented by a combination of dashpots and springs are usually adopted for simulating creep behaviour of composite materials. However, the simplest ones (Maxwell and Kelvin-Voight) are not sufficiently flexible to match experimental data for real materials. The more complex ones, obtained from combination of different Maxwell and Kelvin-Voight models, require instead many parameters and do not allow obtaining inverse Fourier or Laplace transforms in closed form [34, 35]. Therefore, in the 50s of the last century, a viscoelastic stress-strain relation based on fractional derivative has been proposed. The fractional derivative model (FDM) is more flexible and requires a smaller number of parameters, so that their calibration is considerably simpler. The flexibility is due to the order of derivatives which can vary to obtain a constitutive law suitable for the considered material. In 1948 Rabotnov [33] suggested to use fraction-exponential operators that can describe experimental data of real materials with sufficient accuracy and allows one to obtain inverse Laplace transforms analytically [36]. Scott Blair and Coppen $[31,32]$ used fraction-exponential operators for description of viscoelastic properties of materials experimentally (see Rogosin and Mainardi [37]). Fraction-exponential functions in viscoelastic operators were used by many authors last decade. Detailed discussion can be found in the book of Podlubny [38]. Published broad surveys are, for example, [39-41].

The model developed in the present paper is able to describe the creep curve of a PP fibre carefully and it allows obtaining the creep and stress relaxation test response of a fibre composite material in closed form. Moreover, the adopted fraction-exponential operators can be efficiently employed for the homogenization of synthetic FRC by extending the Maxwell scheme developed for elastic composites to viscoelastic behaviour of the constituents (e.g., $[34,42])$.

\section{Materials and Methods}

2.1. Material and Breaking Test. The fibre consists of PP monofilament with a diameter of $0.78 \mathrm{~mm}$ and length of $200 \mathrm{~mm}$. Since the cross section of the fibre is not perfectly circular, the diameter is an average of six measurements: Two measurements in two orthogonal directions in three points of the fibre, namely, at the middle and at both ends. To evaluate elastic Young modulus and tensile strength, tensile tests were performed on four specimens of fibres until their breakage. Each fibre was clamped at its ends and pulled by an electromechanic traction machine under displacement control.

The load cell is a GALDABINI 514262 TYPE TCA, with OUTPUT sensitivity of $2 \mathrm{mV} / \mathrm{V}$. The machine uses a 20 bit A/D converter to acquire the analogical quantities. The resolution of the load cell is $0.002 \mathrm{~N}$ over the entire field 


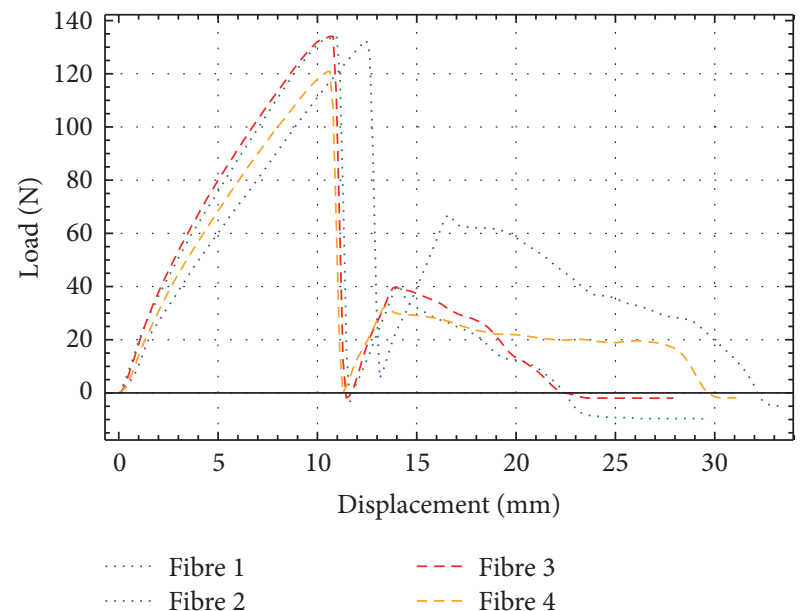

(a)

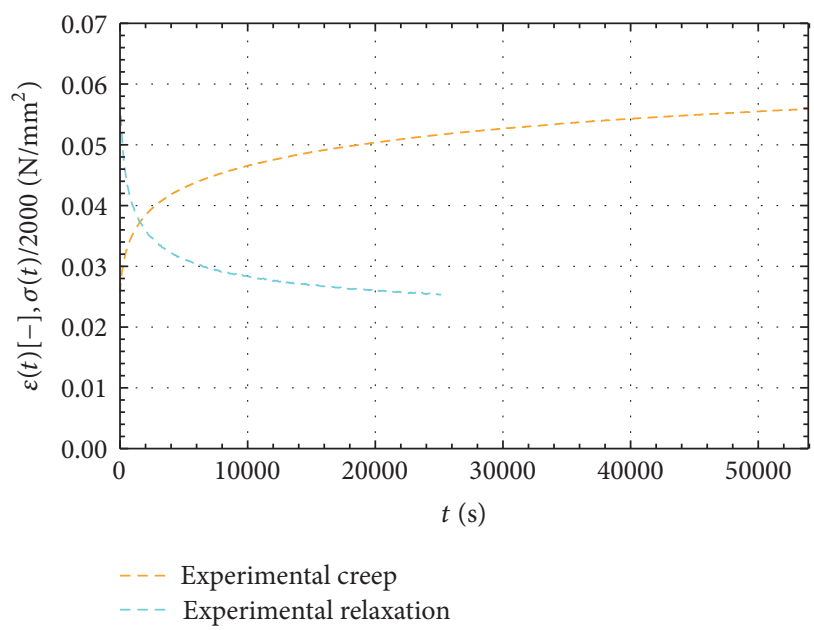

(b)

Figure 1: (a) Tensile tests until breakage on four specimens of fibre: load versus displacement. (b) Experimental curves: creep versus time (dashed orange curve) and stress relaxation versus time (dashed cyan curve: stress scaled by 1/2000).

TABLE 1: Properties of polypropylene fibre.

\begin{tabular}{lc}
\hline Diameter $(\mathrm{mm})$ & 0.78 \\
Tensile strength $\left(\mathrm{N} / \mathrm{mm}^{2}\right)$ & 273.0 \\
Elastic modulus $E_{0}$ at $t=0\left(\mathrm{~N} / \mathrm{mm}^{2}\right)$ & $5.131 \times 10^{3}$ \\
Elastic modulus $E_{\infty}$ at $t \rightarrow \infty\left(\mathrm{N} / \mathrm{mm}^{2}\right)$ & $1.959 \times 10^{3}$ \\
\hline
\end{tabular}

of use, with a capacity of $250 \mathrm{~N}$. The displacement control was performed by the actuator at a rate of $40 \mathrm{~mm} / \mathrm{min}$. The average value of the breakage tensile load is $130.5 \mathrm{~N}$ that occurs approximately at a displacement of $10 \mathrm{~mm}$, namely, at $5 \%$ of strain.

The elastic Young modulus $E_{0}$ (Table 1 ) was determined from the ratio between stress and strain. The stresses and strains were calculated based on the early stage of the experimental load-displacement curve averaged on the four fibres.

The experiments were performed at $25^{\circ} \mathrm{C}$. Since the glass transition temperature of the $\mathrm{PP}$ is approximately $-20^{\circ} \mathrm{C}$ [43], at room temperature PP fibres exhibit a mechanical behaviour corresponding to that of a viscoelastic material in rubbery state, that is, a time-dependent response as shown in Figure 1(b).

Plots of breaking tests are reported in Figure 1(a). In particular, the first peak that can be observed in the curve is due to the breakage of the fibres that occurred on the clamped portion, at which followed a pullout stage until the complete detachment of the fibres. The properties adopted for PP fibre are listed in Table 1. The PP fibre was provided by the Italian company Fili\&Forme ${ }^{\circledR}$ Ltd. and shown in Figure 3.

2.2. Creep and Relaxation Tests. The creep test was carried out on a sample of length $200 \mathrm{~mm}$ subject to a constant tensile force of $60 \mathrm{~N}$ by fixing between two clamps and measuring the displacement of the fibre over the time. The load cell is the same used for the breaking test (see Section 2.1) and the speed to gain $60 \mathrm{~N}$ was performed at a rate of $40 \mathrm{~mm} / \mathrm{min}$. The total duration of the creep test was 15 hours needed for the fibre to achieve a (temporary) stationary value of the deformation (plateau region of the creep curve). However, it should be remarked that the deformation could further increase after 15 hours, since the maximum level of deformation is theoretically achieved at $t \rightarrow \infty$.

It is worth noting that at time longer than $20,000 \mathrm{~s}$ the strain exceeds $5 \%$ but the fibre does not break (Figure 1(b)). Conversely, the fibre under tensile test falls at a strain level equal to $5 \%$ (Figure 1(a)). This is due to the fact that the creep test was performed at a constant load of $60 \mathrm{~N}$, less than onehalf the ultimate tensile load of $130.5 \mathrm{~N}$.

Similarly, the stress relaxation test was carried out on a sample of length $200 \mathrm{~mm}$ subject to a constant displacement of $5 \mathrm{~mm}$ and measuring the tensile force over the time. The speed to gain $5 \mathrm{~mm}$ was performed at a rate of $40 \mathrm{~mm} / \mathrm{min}$. The total duration time was 7 hours, until the force of the fibre was almost stationary. Plots of creep and stress relaxation tests are reported in Figure 1(b).

Both creep and relaxation tests were performed on a single specimen.

The elastic Young modulus $E_{\infty}$ (Table 1) was assessed based on the final stage of the experimental creep and relaxation tests by knowing the imposed constant load and displacement values at which the tests were performed. The assumed values were the averages of the obtained experimental results.

\section{Results and Discussion}

3.1. Analytical Model for Creep and Stress Relaxation Curves. We wanted to fit experimental creep and stress relaxation curves with an analytical model by using fractionexponential kernel that on one side fits carefully experimental data and, at the same time, allows analytical expression for inverse Laplace transform. Let us consider the Boltzmann 
convolution integral that describes the creep strain $\varepsilon(t)$ of a material under a stress $\sigma(t)$ variable over time

$$
\varepsilon(t)=\sigma_{0} \psi(t)+\int_{0}^{t} \dot{\sigma}(\tau) \psi(t-\tau) d \tau,
$$

where $\psi(t)$ is the creep function and $\sigma_{0}=\sigma(0)$ the stress applied at $t=0$. Note that the creep function $\psi(t)$ coincides with the axial strain produced by the constant stress $\sigma(t)=1$. Integrating by parts (1) it follows

$$
\mathcal{\varepsilon}(t)=\sigma(t) \psi_{0}-\int_{0}^{t} \sigma(\tau) \dot{\psi}(t-\tau) d \tau .
$$

By following [34], we write (2) in the form

$$
\varepsilon(t)=\frac{1}{E_{0}}\left[\sigma(t)-\lambda \int_{0}^{t} R_{\alpha}(\beta-\lambda, t-\tau) \sigma(\tau) d \tau\right],
$$

where

$$
\begin{aligned}
R_{\alpha}(\beta-\lambda, t-\tau) & =(t-\tau)^{\alpha} \sum_{n=0}^{\infty} \frac{(\beta-\lambda)^{n}(t-\tau)^{n(1+\alpha)}}{\Gamma[(n+1)(1+\alpha)]}, \\
\psi_{0} & =\frac{1}{E_{0}}, \\
\lambda & =\frac{E_{0}-E_{\infty}}{E_{0}} \beta,
\end{aligned}
$$

where $R_{\alpha}$ is the Rabotnov function that allows using fractionexponential kernel in viscoelastic operators, and $E_{0}, E_{\infty}$ are the elastic Young modulus at $t=0, t \rightarrow \infty$, respectively. A constant stress $\sigma(t)=\sigma_{0}$ is then assumed in order to simulate the creep test, so that

$$
\begin{aligned}
& \int_{0}^{t} R_{\alpha}(\beta-\lambda, t-\tau) \sigma(\tau) d \tau \\
& \quad=\sigma_{0} \sum_{n=0}^{\infty} \frac{(\beta-\lambda)^{n}}{\Gamma[(n+1)(1+\alpha)]} \int_{0}^{t}(t-\tau)^{n(1+\alpha)+\alpha} d \tau \\
& \quad=\sigma_{0} \sum_{n=0}^{\infty} \frac{(\beta-\lambda)^{n}(t-\tau)^{(n+1)(1+\alpha)}}{\Gamma[(n+1)(1+\alpha)+1]},
\end{aligned}
$$

where the property of Euler gamma function $\Gamma(z+1)=z \Gamma(z)$ has been used.

By shifting the index $m=n+1$, it follows

$$
\begin{aligned}
& \frac{\sigma_{0}}{\beta-\lambda} \sum_{n=0}^{\infty} \frac{(\beta-\lambda)^{n+1} t^{(n+1)(1+\alpha)}}{\Gamma[(n+1)(1+\alpha)+1]} \\
& =\frac{\sigma_{0}}{\beta-\lambda}\left\{\sum_{m=0}^{\infty} \frac{(\beta-\lambda)^{m} t^{m(1+\alpha)}}{\Gamma[m(1+\alpha)+1]}-1\right\} \\
& =\frac{\sigma_{0}}{\beta-\lambda}\left\{M_{1+\alpha}\left[(\beta-\lambda) t^{1+\alpha}\right]-1\right\},
\end{aligned}
$$

where the Mittag-Leffler function,

$$
M_{a}(z)=\sum_{m=0}^{\infty} \frac{z^{m}}{\Gamma[m a+1]},
$$

has been introduced. Therefore, (3) becomes

$$
\varepsilon(t)=\frac{\sigma_{0}}{E_{0}}\left[1-\frac{\lambda}{\beta-\lambda}\left\{M_{1+\alpha}\left[(\beta-\lambda) t^{1+\alpha}\right]-1\right\}\right] .
$$

By using the following properties of the Mittag-Leffler function

$$
\begin{aligned}
& \lim _{t \rightarrow 0} M_{1+\alpha}\left[(\beta-\lambda) t^{1+\alpha}\right]=1, \\
& \lim _{t \rightarrow \infty} M_{1+\alpha}\left[(\beta-\lambda) t^{1+\alpha}\right]=0,
\end{aligned}
$$

then, from (8) and (9), it follows

$$
\begin{gathered}
\varepsilon_{0}=\lim _{t \rightarrow 0} \varepsilon(t)=\frac{\sigma_{0}}{E_{0}}, \\
\varepsilon_{\infty}=\lim _{t \rightarrow \infty} \varepsilon(t)=\frac{\sigma_{0}}{E_{\infty}},
\end{gathered}
$$

where $\varepsilon_{0}$ and $\varepsilon_{\infty}$ are the creep strains at $t=0, t \rightarrow \infty$, respectively.

Again, let us consider the Boltzmann convolution integral that describes the relaxation stress $\sigma(t)$ of a material under a strain variable over time $\varepsilon(t)$.

$$
\sigma(t)=\varepsilon_{0} \phi(t)+\int_{0}^{t} \dot{\varepsilon}(\tau) \phi(t-\tau) d \tau,
$$

where $\phi(t)$ is the relaxation function and $\varepsilon_{0}=\varepsilon(0)$ the strain applied at $t=0$. The relaxation function $\phi(t)$ coincides with the axial stress produced by the constant strain $\varepsilon(t)=1$. Integrating by parts (11) it follows

$$
\sigma(t)=\varepsilon(t) \phi_{0}-\int_{0}^{t} \varepsilon(\tau) \dot{\phi}(t-\tau) d \tau .
$$

By following [34], (12) can be written in the form

$$
\begin{aligned}
\sigma(t) & =E_{0}\left[\varepsilon(t)+\lambda \int_{0}^{t} R_{\alpha}(\beta, t-\tau) \varepsilon(\tau) d \tau\right], \\
\phi_{0} & =E_{0} .
\end{aligned}
$$

Assuming a constant strain $\varepsilon(t)=\varepsilon_{0}$ in order to simulate the stress relaxation test and following the way to find (8), (13) becomes

$$
\sigma(t)=E_{0} \varepsilon_{0}\left\{1+\frac{E_{0}-E_{\infty}}{E_{0}}\left[M_{1+\alpha}\left(\beta t^{1+\alpha}\right)-1\right]\right\} .
$$

For the properties of the Mittag-Leffler function, from (14) it follows

$$
\begin{gathered}
\sigma_{0}=\lim _{t \rightarrow 0} \sigma(t)=E_{0} \varepsilon_{0}, \\
\sigma_{\infty}=\lim _{t \rightarrow \infty} \sigma(t)=E_{\infty} \varepsilon_{0},
\end{gathered}
$$

where $\sigma_{0}$ and $\sigma_{\infty}$ are the stress relaxation at $t=0, t \rightarrow \infty$, respectively.

The equations (8) and (14) represent the model by means of the fact that it was possible to fit accurately the experimental creep and stress relaxation tests for suitable values of parameters $\alpha$ and $\beta$. The plots of experimental and analytical creep and stress relaxation curves are reported in Figure 2(a) and the corresponding parameters $\alpha$ and $\beta$ are reported in Table 2 . 


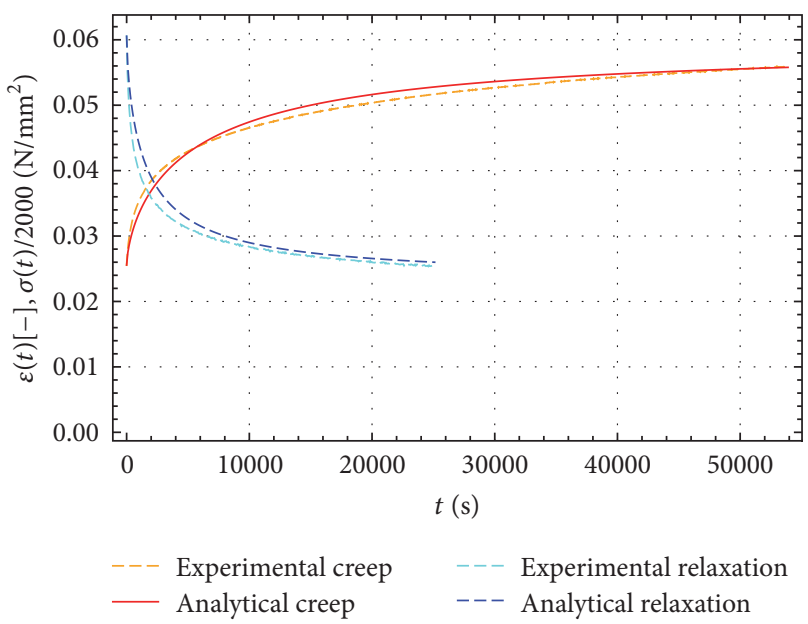

(a)

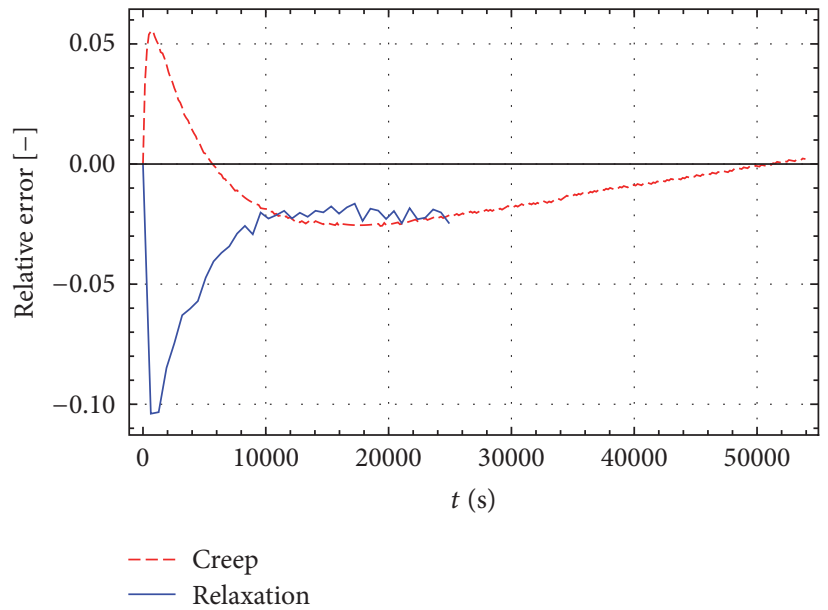

(b)

Figure 2: (a) Experimental creep curve (dashed orange curve) and analytical creep curve according to (8) (solid red curve). Experimental stress relaxation curve (dashed cyan curve) and analytical stress relaxation curve according to (14) (dashed blue curve) both scaled by $1 / 2000$. (b) Relative error between experimental data on creep and relaxation and their model given by (8) and (14).

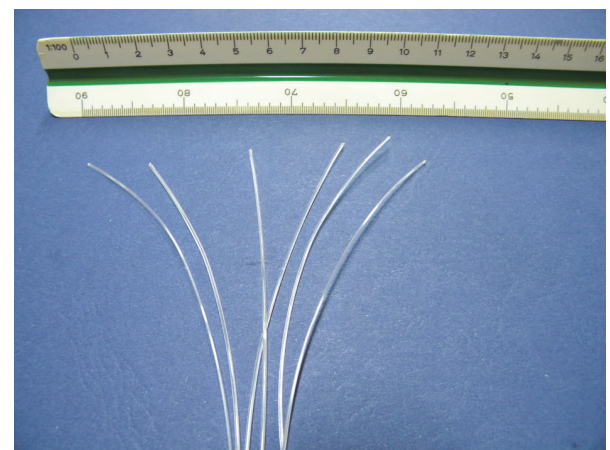

FIGURE 3: Polypropylene fibres used for tensile until breakage and creep and stress relaxation tests [3].

TABle 2: Parameters $\alpha$ and $\beta$ that fit experimental creep and relaxation curves using the model given by (8) and (14).

\begin{tabular}{lc}
\hline$\alpha$ (dimensionless) & $-1 / 3$ \\
$\beta\left(\mathrm{s}^{-(1+\alpha)}\right)$ & $-1 /(100 \sqrt{3})$ \\
\hline
\end{tabular}

\section{Conclusions}

In the present paper we carried out a tensile test until breakage and a creep and stress relaxation test on a PP fibre and fitted these experimental tests by using a viscoelastic model based on fractional-exponential kernel. The curves plotted in Figure 2(a) show that the theoretical model closely fits the experimental results. The relative error, showing the deviation between the experimental data and the theoretical prediction, is plotted in Figure 2(b). It shows a maximum relative error of $5 \%$ and $10 \%$, respectively, for creep and relaxation, occurring in a very small portion of the curves.
Conversely to complex viscoelastic models based on combinations of Maxwell and Kelvin-Voight schemes, the proposed model requires the calibration of only two parameters ( $\alpha$ and $\beta$ ) to simulate creep and relaxation phenomena [35]. Moreover, for simple load or displacement histories, it allows calculating straightforwardly the stress and strain fields based on the calculation of the inverse Laplace transform in closed form. Note also that the Rabotnov function generally allows fitting the experimental data better than other conventional schemes based on the combination of springs and dashpots [34].

It has been proved that the model here adopted is able to match carefully experimental data obtained for PP fibres. In a forthcoming work, the present investigation will be extended in order to take into account the effects induced by thermal variations acting on FRC elements (for the thermodynamic aspects of thermoelasticity, see, e.g., [44]).

\section{Competing Interests}

The author declares that they have no competing interests.

\section{References}

[1] P. K. Mallick, Fiber Reinforced Composites-Materials, Manufacturing, and Design, CRC Press, New York, NY, USA, 2008.

[2] L. Lanzoni, A. Nobili, E. Radi, and A. Sorzia, "Axisymmetric loading of an elastic-plastic plate on a general two-parameter foundation," Journal of Mechanics of Materials and Structures, vol. 10, no. 4, pp. 459-479, 2015.

[3] P. Di Maida, E. Radi, C. Sciancalepore, and F. Bondioli, "Pullout behavior of polypropylene macro-synthetic fibers treated with nano-silica," Construction and Building Materials, vol. 82, pp. 39-44, 2015.

[4] R. Barretta, L. Feo, and R. Luciano, "Some closed-form solutions of functionally graded beams undergoing nonuniform torsion," Composite Structures, vol. 123, pp. 132-136, 2015. 
[5] R. Luciano and J. R. Willis, "Hashin-Shtrikman based FE analysis of the elastic behaviour of finite random composite bodies," International Journal of Fracture, vol. 137, no. 1, pp. 261273, 2006.

[6] A. Nobili, L. Lanzoni, and A. M. Tarantino, "Experimental investigation and monitoring of a polypropylene-based fiber reinforced concrete road pavement," Construction and Building Materials, vol. 47, pp. 888-895, 2013.

[7] E. S. Bernard, "Design of fibre reinforced shotcrete linings with macro-synthetic fibres," in Proceedings of the 11th International Conference on Shotcrete for Underground Support, F. Amberg and K. F. Garshol, Eds., vol. P11 of Engineering Conferences International Symposium Series, 2009, http://dc.engconfintl.org/shotcrete/14.

[8] L. Lanzoni, A. Nobili, and A. M. Tarantino, "Performance evaluation of a polypropylene-based draw-wired fibre for concrete structures," Construction and Building Materials, vol. 28, no. 1, pp. 798-806, 2012.

[9] G. Xotta, G. Mazzucco, V. A. Salomoni, C. E. Majorana, and K. J. Willam, "Composite behavior of concrete materials under high temperatures," International Journal of Solids and Structures, vol. 64-65, pp. 86-99, 2015.

[10] V. A. Salomoni, C. E. Majorana, G. M. Giannuzzi, and A. Miliozzi, "Thermal-fluid flow within innovative heat storage concrete systems for solar power plants," International Journal of Numerical Methods for Heat and Fluid Flow, vol. 18, no. 7-8, pp. 969-999, 2008.

[11] V. A. Salomoni, C. E. Majorana, B. Pomaro, G. Xotta, and F. Gramegna, "Macroscale and mesoscale analysis of concrete as a multiphase material for biological shields against nuclear radiation," International Journal for Numerical and Analytical Methods in Geomechanics, vol. 38, no. 5, pp. 518-535, 2014.

[12] G. Dinelli, G. Belz, C. E. Majorana, and B. A. Schrefler, "Experimental investigation on the use of fly ash for lightweight precast structural elements," Materials and Structures, vol. 29, no. 194, pp. 632-638, 1996.

[13] A. M. Tarantino, "On the finite motions generated by a mode I propagating crack," Journal of Elasticity, vol. 57, no. 2, pp. 85$103,1999$.

[14] A. M. Tarantino, "Crack propagation in finite elastodynamics," Mathematics and Mechanics of Solids, vol. 10, no. 6, pp. 577-601, 2005.

[15] A. M. Tarantino, "The singular equilibrium field at the notch-tip of a compressible material in finite elastostatics," Zeitschrift für Angewandte Mathematik und Physik, vol. 48, no. 3, pp. 370-388, 1997.

[16] A. M. Tarantino, "On extreme thinning at the notch tip of a neoHookean sheet," The Quarterly Journal of Mechanics and Applied Mathematics, vol. 51, no. 2, pp. 179-190, 1998.

[17] A. M. Tarantino, "Nonlinear fracture mechanics for an elastic Bell material," The Quarterly Journal of Mechanics and Applied Mathematics, vol. 50, no. 3, pp. 435-456, 1997.

[18] M. Di Prisco, G. Plizzari, and L. Vandewalle, "Fibre reinforced concrete: new design perspectives," Materials and Structures, vol. 42, no. 9, pp. 1261-1281, 2009.

[19] E. Radi and P. Di Maida, "Analytical solution for ductile and FRC plates on elastic ground loaded on a small circular area," Journal of Mechanics of Materials and Structures, vol. 9, no. 3, pp. 313-331, 2014.

[20] L. Dezi and A. M. Tarantino, "Time dependent analysis of concrete structures with variable structural system," ACI Materials Journal, vol. 88, no. 3, pp. 320-324, 1991.
[21] L. Dezi, G. Menditto, and A. M. Tarantino, "Viscoelastic heterogeneous structures with variable structural system," Journal of Engineering Mechanics, vol. 119, no. 2, pp. 238-250, 1993.

[22] L. Dezi, G. Menditto, and A. M. Tarantino, "Homogeneous structures subjected to repeated structural system changes," Journal of Engineering Mechanics, vol. 116, no. 8, pp. 1723-1732, 1990.

[23] R. Barretta, L. Feo, and R. Luciano, "Torsion of functionally graded nonlocal viscoelastic circular nanobeams," Composites Part B: Engineering, vol. 72, pp. 217-222, 2015.

[24] R. Barretta, L. Feo, R. Luciano, and F. Marotti de Sciarra, "A gradient Eringen model for functionally graded nanorods," Composite Structures, vol. 131, pp. 1124-1131, 2015.

[25] R. Barretta, L. Feo, R. Luciano, and F. Marotti de Sciarra, "Variational formulations for functionally graded nonlocal Bernoulli-Euler nanobeams," Composite Structures, vol. 129, pp. 80-89, 2015.

[26] V. Salomoni, G. Mazzucco, C. Pellegrino, and C. Majorana, "Three-dimensional modelling of bond behaviour between concrete and FRP reinforcement," Engineering Computations, vol. 28, no. 1, pp. 5-29, 2011.

[27] A. Caporale, L. Feo, R. Luciano, and R. Penna, "Numerical collapse load of multi-span masonry arch structures with FRP reinforcement," Composites Part B: Engineering, vol. 54, no. 1, pp. 71-84, 2013.

[28] A. Caporale and R. Luciano, "Limit analysis of masonry arches with finite compressive strength and externally bonded reinforcement," Composites Part B: Engineering, vol. 43, no. 8, pp. 3131-3145, 2012.

[29] P. Bisegna and R. Luciano, "Bounds on the overall properties of composites with debonded frictionless interfaces," Mechanics of Materials, vol. 28, no. 1-4, pp. 23-32, 1998.

[30] A. Apuzzo, R. Barretta, and R. Luciano, "Some analytical solutions of functionally graded Kirchhoff plates," Composites Part B: Engineering, vol. 68, pp. 266-269, 2015.

[31] G. W. Scott-Blair and F. M. V. Coppen, "The subjective judgement of the elastic and plastic properties of soft bodies; the 'differential thresholds' for viscosities and compression moduli," Proceedings of the Royal Society B: Biological Sciences, vol. 128, no. 850, pp. 109-125, 1939.

[32] G. W. Scott Blair and F. M. V. Coppen, "The estimation of firmness in soft materials," The American Journal of Psychology, vol. 56, no. 2, pp. 234-246, 1943.

[33] Yu. N. Rabotnov, "Equilibrium of an elastic medium with aftereffect," Journal of Applied Mathematics and Mechanics, vol. 12, pp. 53-62, 1948 (Russian).

[34] I. Sevostianov, V. Levin, and E. Radi, "Effective properties of linear viscoelastic microcracked materials: application of Maxwell homogenization scheme," Mechanics of Materials, vol. 84, pp. 28-43, 2015.

[35] F. Di Paola and F. P. Pinnola, Calcolo Frazionario \& Viscoelasticità, Dipartimento di Ingegneria Civile Ambientale e Aerospaziale, Università degli Studi di Palermo, Palermo, Italy, http://wwwl.unipa.it/fabio.bagarello/didattica/Di\%20Paola\% 20e\%20Pinnola_calcolo\%20frazionario.pdf.

[36] Yu. N. Rabotnov, Elements of Hereditary Solid Mechanics, Mir, Moscow, Russia, 1977.

[37] S. Rogosin and F. Mainardi, "George William Scott Blair-the pioneer of fractional calculus in rheology," Communications in Applied and Industrial Mathematics, vol. 6, no. 1, article e-481, 2014. 
[38] I. Podlubny, Fractional Differential Equations, Academic Press, New York, NY, USA, 1998.

[39] F. Mainardi, "Fractional calculus: some basic problems in continuum and statistical mechanics," in Fractals and Fractional Calculus in Continuum Mechanics, A. Carpinteri and F. Mainardi, Eds., Springer, Vienna, Austria, 1997.

[40] F. Mainardi, "Applications of fractional calculus in mechanics," in Transform Methods and Special Functions, Varna '96, P. Rusev, I. Dimovski, and V. Kiryakova, Eds., SCT, Singapore, 1997.

[41] Yu. A. Rossikhin and M. V. Shitikova, "Applications of fractional calculus to dynamic problems of linear and nonlinear hereditary mechanics of solids," Applied Mechanics Reviews, vol. 50, no. 1, pp. 15-67, 1997.

[42] F. Greco and R. Luciano, "A theoretical and numerical stability analysis for composite micro-structures by using homogenization theory," Composites Part B: Engineering, vol. 42, no. 3, pp. 382-401, 2011.

[43] B. Ellis and R. Smith, Polymers A Property Database, CRC Press, New York, NY, USA, 2nd edition, 2009.

[44] F. Marotti de Sciarra and M. Salerno, "On thermodynamic functions in thermoelasticity without energy dissipation," European Journal of Mechanics A: Solids, vol. 46, pp. 84-95, 2014. 


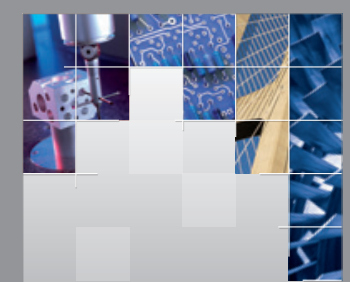

\section{Enfincering}
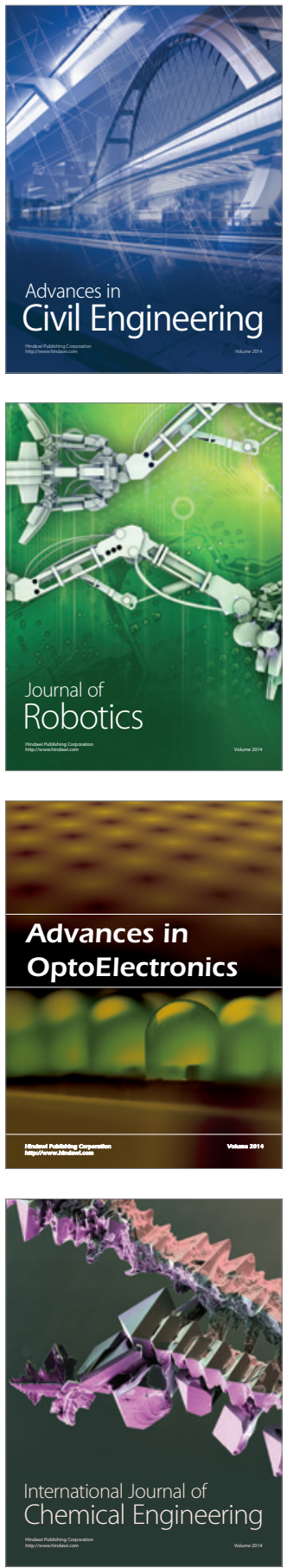

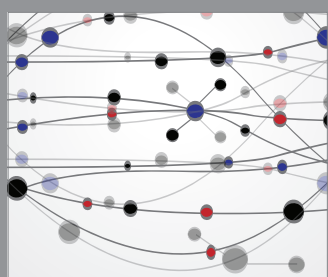

The Scientific World Journal

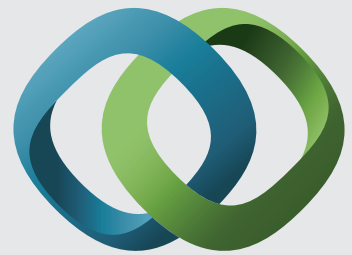

\section{Hindawi}

Submit your manuscripts at

http://www.hindawi.com
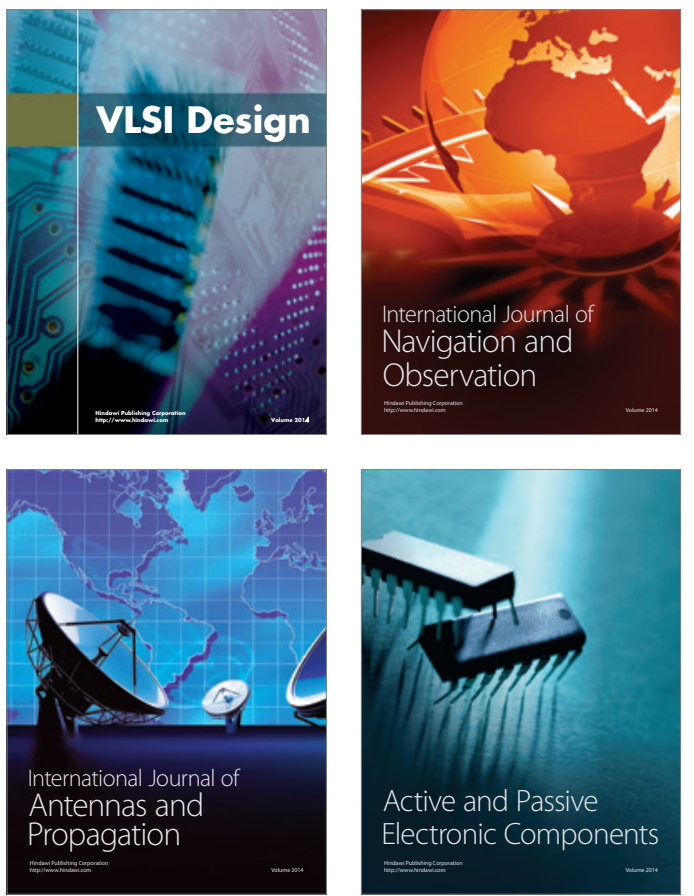
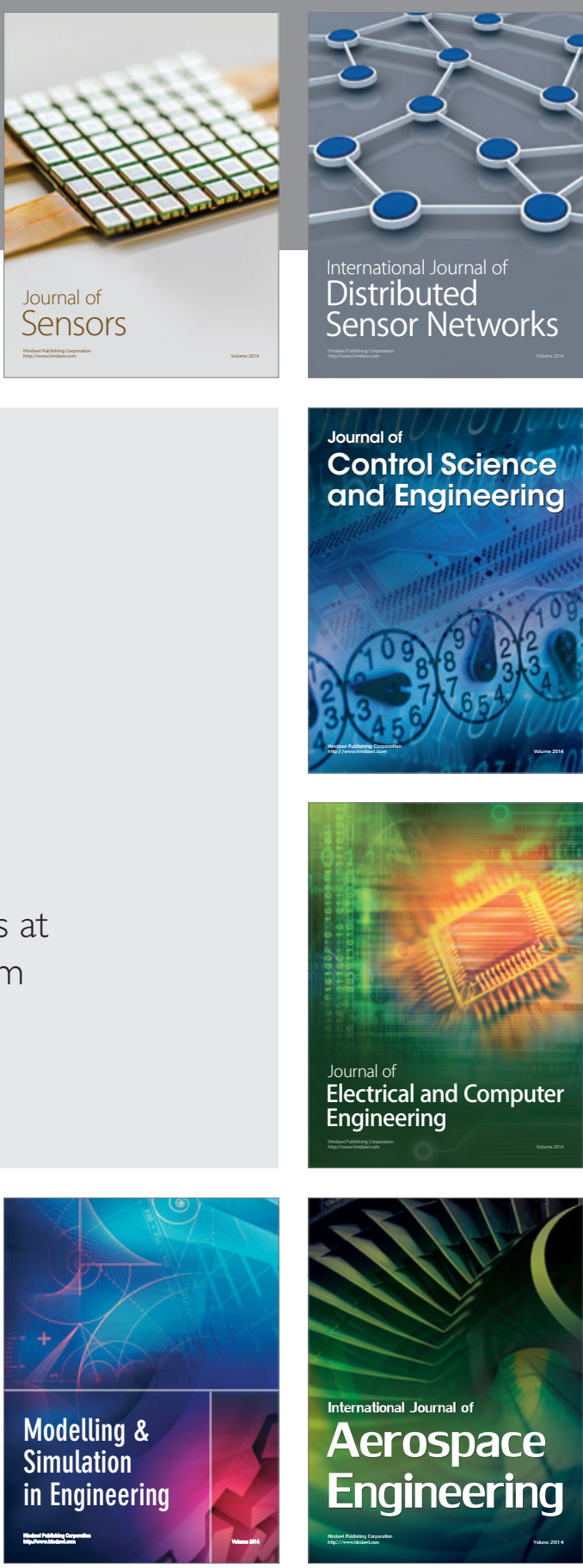

International Journal of

Distributed

Sensor Networks

Journal of

Control Science

and Engineering
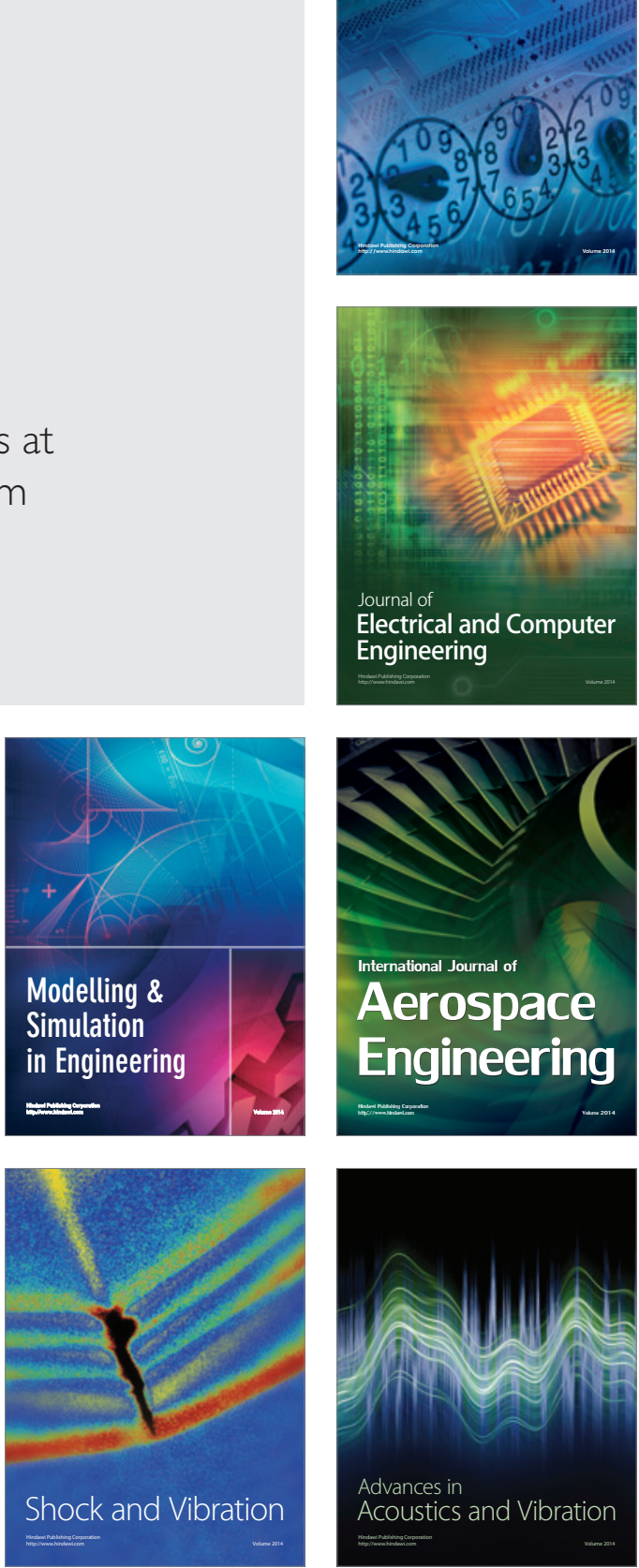